\title{
Multi-Scale Turbulence Model in Simulation of Supersonic Crossflow
}

\author{
Ez Hassan ${ }^{*}$ and Hikaru Aono ${ }^{\dagger}$ \\ University of Michigan, Ann Arbor, MI \\ John Boles \\ Taitech, Inc. ,Beavercreek, $\mathrm{OH}$ \\ Douglas Davis ${ }^{\S}$ \\ Air Force Research Laboratory, Fairborn, $\mathrm{OH}$ \\ Wei Shyy ${ }^{* * \dagger}$ \\ University of Michigan, Ann Arbor, MI \\ The Hong Kong University of Science and Technology, Kowloon, Hong Kong
}

Hybrid Reynolds-averaged Navier-Stokes/large eddy simulation modeling approaches are convenient for flows where parts of the flowfield can be solved well with Reynolds-averaged Navier Stokes (RANS) models and large eddy simulations (LES) can be used in areas where the flow contains complex turbulent. This work is aimed at assessing the capability of a multi-scale hybrid RANS/LES computational modeling approach in solving an Ethylene injection case into supersonic crossflow. This approach allows for the computation to have a large range of varying grid size, geometry and flow regimes as it smoothly varies the eddy viscosity to resolve turbulent structures consistent with the grid. Fuel concentration and eddy viscosity contours reveal physical turbulent structures that increase with grid refinement. Predictions of the time averaged fuel mole fraction from the hybrid model are improved over RANS simulations when compared to experimental measurements. Turbulent fluctuations are collected to estimated resolved mass diffusion and momentum eddy viscosities. The resolved mass and momentum diffusion show large variations in turbulent Schmidt number.

\section{Nomenclature}

$C_{p} \quad=$ heat capacity at constant pressure

$C_{s} \quad=$ Smagorinsky constant

$D \quad=$ injector inner diameter

$D_{i_{m}} \quad=$ diffusion vector for species $\mathrm{m}$

$E=$ total specific energy of the mixture

$H \quad=$ total specific enthalpy of the mixture

$F_{2} \quad=$ second blending function, Menter SST

$f_{d} \quad=$ filter function

${ }^{*}$ PhD Candidate, Aerospace Engineering Department

${ }^{\dagger}$ Postdoctoral Research Fellow, Aerospace Engineering Department

* Research Scientist, AFRL/RZAS

$\S$ Aerospace Engineer, AFRL/RZAS

** Clarence L. "Kelly" Johnson Collegiate Professor, Aerospace Engineering Department

${ }^{\dagger}$ Chair Professor , Mechanical Engineering at HKUST

American Institute of Aeronautics and Astronautics 


$\begin{array}{ll}h_{m} & =\text { species specific enthalpy } \\ k & =\text { turbulent specific kinetic energy } \\ L_{G} & =\text { length scale based on cell size } \\ L_{T} & =\text { measure of turbulence length scale } \\ \mathrm{NS} & =\text { total number of species } \\ p & =\text { pressure } \\ P r_{t} & =\text { turbulent Prandtl number } \\ q_{i} & =\text { heat transfer vector } \\ \mathrm{S} & =\text { absolute strain rate } \\ S c_{t} & =\text { turbulent Schmidt number } \\ T & =\text { mixture temperature } \\ u_{i} & =\text { mixture velocity } \\ Y_{m} & =\text { mole fraction of species m } \\ \mu_{t} & =\text { dynamic eddy viscosity } \\ v_{t} & =\text { kinematic eddy viscosity } \\ \dot{\dot{\omega}} & =\text { average chemistry source term } \\ \Omega & =\text { absolute vorticity } \\ \rho & =\text { mixture density } \\ \tau_{i j} & =\text { stress tensor } \\ (\sim) & =\text { filter operator } \\ (-) & =\text { average operator } \\ \mathrm{R} & =\text { superscript for Reynolds terms } \\ \text { Res } & =\text { subscript for quantities calculated from resolved field }\end{array}$

\section{Introduction}

Upersonic crossflow analysis is needed to understand the physics behind supersonic combustion occurring in

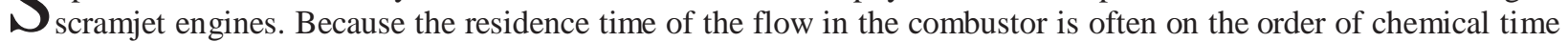
scales, it is of utmost importance for the fuel and oxidizer to be mixed quickly. It is necessary to gain better understanding of the effect of different injection configuration and combustor geometries on the injection process in order to achieve desirable designs for scramjet engines.

Several experimental studies have been done to understand the mechanisms of the jet mixing in a supersonic crossflow using velocity measurements ${ }^{1}$, time-averaged wall-pressure measurements ${ }^{2}$ and temporally resolved flow visualizations and mixing characteristics with nonreactive $e^{3,4}$ and combustible gaseous jets ${ }^{5}$. The results have presented the major flow phenomena, such as the prominent bow shock, the Mach disk, the counter-rotating vortices in the injection plume, and the wake and vortex regions beneath the injection plume. Even though measurement technologies have advanced significantly, experimental data is still limited due to cost, complexity of the flow, and reliability of the measured data. Therefore, computational studies have been showing an attractive alternative to understand the flow physics and augment the data of sonic flow injection into supersonic flowfields.

Reynolds-averaged Navier-Stokes (RANS) based approaches have shown some success in understanding the mechanics of supersonic crossflows. Tam et al. ${ }^{6}$ used RANS based methods with Menter SST ${ }^{7}$, Menter BSL ${ }^{7}$, and Wilcox $k-\omega^{8}$ turbulence models to simulate the experiments of Gruber et al. ${ }^{4,9,10}$. It was shown that the Wilcox $k-\omega$ model performed the best of all three; however, in all the models tested the fuel penetration height was over predicted by up to $25 \%$. Palekar et al. ${ }^{11}$ obtained better correlations with penetration heights with a 4.7 million cell grid using the commercial CFD code GASP. The Wilcox $k-\omega$ turbulence model was also utilized for turbulence closure. Coarser grids did not sufficiently resolve the flow, and spanwise fuel penetration significantly deviated from the experiment. Maddalena et al. ${ }^{12}$ used the Wilcox $k$ - $\omega$ turbulence model to simulate an aeroramp injection scheme as well as transverse injection of sonic helium into air. Total pressure loss was shown to be greater than of normal injection configuration with the computational results not correlating well with the experiment.

As stated by many researchers ${ }^{13-15}$, there are some obvious limitations to RANS models when applied to unsteady problems because they tend to be overly dissipative (predicting higher eddy viscosity and damping the unsteady motion of the fluid). The crossflow problem usually involves large scale unsteady turbulent structures, density gradients, and shock boundary layer interactions as evident by experimental findings ${ }^{4,9,10}$. These phenomena are difficult to capture correctly with standard RANS approaches and may benefit from methods capable of 
resolving turbulent structures such as Direct Numerical Simulation (DNS) or Large Eddy Simulation (LES). LES is capable of capturing the large scale turbulent structures, and while not as computationally expensive as DNS, it is impractical for use in the supersonic crossflow problem because a prohibitively large number of grid points must be used to resolve the boundary layer. Recently, Kawai and Lele ${ }^{16}$ conducted an LES of sonic injection into a supersonic crossflow and showed key physics of the jet mixing in supersonic crossflow such as clockwise and counterclockwise rotating strong longitudinal vortices form two groups of counter-rotating vortices, a pair of Ushaped counter rotating vortices. To reduce the expense of the computations, the Reynolds number was lowered by a factor of six, relative to the experiment ${ }^{1}$ but the boundary layer thickness upstream of jet injection is matched with the experiment ${ }^{1}$.

Detached eddy simulation (DES) ${ }^{15}$ and Hybrid RANS/LES methods, where RANS is used at the wall boundary and LES is used elsewhere, has shown a great promise in the solution of supersonic crossflow problem. Peterson et al. ${ }^{17}$ used a DES model based on Spalart-Allmaras one equation turbulence model to simulate supersonic cross flow experiments conducted at Virginia Polythechnic University. DES compared favorably to the experiment with results superior to RANS. Boles et al. ${ }^{18-20}$ simulated Gruber et al. ${ }^{4,9,10}$ air and helium injection cases as well as Ethylene injection case conducted at Air Force Research Laboratory (AFRL) and reported by Lin et al. ${ }^{21}$. In all cases time averaged Hybrid results were superior to RANS quantitatively and qualitatively when compared to experimental injectant distribution.

In the original DES Method, the transition to LES was based solely on grid spacing, and sometimes it resulted in the LES mode turned on in the boundary layer in areas where it cannot effectively resolve the turbulent length scale $^{22}$. Also DES faced a log layer mismatch in turbulence quantities due to transition from RANS to LES. These problems were later alleviated by involving the RANS eddy viscosity in the definition of the length scale to insure LES is used only when the turbulence length scale is large enough to be resolved this was called Delayed DES (DDES) ${ }^{22}$. Also a blending function was used to smooth the transition between RANS and LES to prevent the log layer mismatch, this was referred to as Improved DDES (IDDES) ${ }^{23}$. The Limited Numerical Scales (LNS) model ${ }^{23}$, also blends RANS and LES by using a latency factor to reduce the production and destruction terms of the turbulent kinetic energy and limit the eddy viscosity used in the momentum equation.

Above-mentioned Hybrid simulations while computationally cheaper than LES, still require a large number computational cells because they constrain their RANS simulation to a thin layer near the wall. Also implementation of such models is usually difficult especially when the subgrid model is not related to the RANS turbulence model. ${ }^{20}$

In this effort we propose the use of multi-scale turbulence modeling ${ }^{24}$ in which two turbulence length scales are used as opposed to only one for the two equation models. The multi-scale model is very easy to implement to any two equation model (In this study we select Menter SST turbulence model ${ }^{7}$ ) by defining a turbulent length scale and a blending function. The eddy viscosity is smoothly varied based on the ratio of the turbulence length scale to the cell size. Therefore there is no sharp transition between RANS and LES and no restriction on where the transition should occur. This allows the use of any grid resolution with the finer grids simply capable of resolving more turbulent eddies. Because of the smooth nature of the model we are able to use adaptive grid and expect smooth solutions at the adaptation interface. In the supersonic crossflow problem this will be especially useful when using $3 \mathrm{D}$ grids that are only fine in the plume region and in regions where complex flow phenomena occur.

The test case used is based on experiments conducted at AFRL and reported by Lin et $\mathrm{al}^{21}$ for injection of sonic Ethylene into Mach 2 air. Injection was conducted at either 90 or 30 degrees with momentum ratios varying from 0.25 to 1.5. We focus here on the normal injection with momentum ratio of 0.5 . This particular case has been simulated with remarkable success with Hybrid RANS/LES methods combined with unsteady inflow boundary conditions to artificially induce fluctuations ${ }^{18,20,25}$. In this study, we examine the fidelity of the multi scale model at different grid resolutions and its capability to resolve turbulent flow structures correctly. We compare time-averaged ethylene mole fraction to the experimental measurements and to RANS solution with varying turbulent Schmidt number $\left(\mathrm{Sc}_{\mathrm{t}}\right)$. We also collect turbulent statistics to estimate turbulence kinetic energy and mass and momentum eddy viscosity based on the resolved field. We then look at the variation of the turbulent Schmidt number $\left(\mathrm{Sc}_{\mathrm{t}}\right)$ predicted by resolved quantities.

\section{Governing Equations and Computational Modeling Approaches}

A density-based, finite volume code, Loci-Chem ${ }^{26,27}$, is utilized in this study. The code is capable of handling mixed element type unstructured grids. The convective fluxes are based on Roe's flux difference splitting ${ }^{28}$. Both convective and diffusive fluxes are evaluated to second order accuracy. Menter's Shear Stress Transport ${ }^{7}$ (SST) model is used for turbulence closure along with multi scale treatment. 


\section{A. Multi-Scale Turbulence Treatment:}

With the concept of eddy viscosity used in either a standard two-equation RANS approach or the Smagorinsky subgrid model in LES, both filtered and averaged mass, momentum, and energy equations yield an identical mathematical form,

$$
\begin{aligned}
& \frac{\partial \bar{\rho} \tilde{Y}_{m}}{\partial t}+\frac{\partial}{\partial x_{i}}\left(\bar{\rho} \tilde{u}_{i}\right)=\frac{\partial}{\partial x_{i}}\left(D_{i_{m}}+D_{i_{m}}^{R}\right)+\overline{\dot{\omega}} \\
& \frac{\partial}{\partial t}\left(\bar{\rho} \tilde{u}_{i}\right)+\frac{\partial}{\partial x_{i}}\left(\bar{\rho} \tilde{u}_{i} \tilde{u}_{j}\right)=-\frac{\partial \bar{p}}{\partial x_{i}}+\frac{\partial}{\partial x_{j}}\left(\tau_{i j}+\tau^{R}{ }_{i j}\right) \\
& \frac{\partial}{\partial t}(\bar{\rho} \tilde{E})+\frac{\partial}{\partial x_{i}}\left(\bar{\rho} \tilde{H} \tilde{u}_{j}\right)=\frac{\partial}{\partial x_{j}}\left[\tilde{u}_{j}\left(\tau_{i j}+\tau^{R}{ }_{i j}\right)-\left(q_{j}+q^{R}{ }_{j}\right)\right]
\end{aligned}
$$

Where the Reynolds terms are modeled as follows:

$$
\begin{aligned}
& D_{i_{m}}^{R} \approx-\frac{\mu_{t}}{S c_{t}} \frac{\partial \tilde{Y}_{m}}{\partial x_{j}} \\
& \tau_{i j} \approx \mu_{t}\left(\frac{\partial \tilde{u}_{i}}{\partial x_{j}}+\frac{\partial \tilde{u}_{j}}{\partial x_{i}}-\frac{2}{3} \frac{\partial \tilde{u}_{k}}{\partial x_{k}}\right) \\
& q_{j} \approx-\frac{\mu_{t} C_{p}}{\operatorname{Pr}_{t}} \frac{\partial \tilde{T}}{\partial x_{j}}-\sum_{m=1}^{N S} h_{m} \frac{\mu_{t}}{S C_{t}} \frac{\partial \tilde{Y}_{m}}{\partial x_{j}}
\end{aligned}
$$

The variables in Eqs. (1) and (2) are shown in the filtered form, however they could represent averaged quantities in which case the value of the eddy viscosity would be produced via a two equation turbulence model. They could also be instantaneous variables when the eddy viscosity is set to zero. When they are filtered, a Smagorinsky subgrid eddy viscosity is defined as follows:

$$
\begin{aligned}
& \mu_{t}=\rho C_{s} L_{G}{ }^{2} S \\
& S=\sqrt{\frac{\partial \tilde{u_{i}}}{\partial x_{j}} \frac{\partial \tilde{u_{j}}}{\partial x_{i}}+\frac{\partial \tilde{u_{i}}}{\partial x_{j}} \frac{\partial \tilde{u_{i}}}{\partial x_{j}}-\frac{2}{3}\left(\frac{\partial \tilde{u_{i}}}{\partial x_{i}}\right)^{2}}
\end{aligned}
$$

Assuming that we average the result of each model, Eqs.(1) and (2) combined with the averaging procedure, become a single mathematical formulation in which we input an eddy viscosity and output averaged quantities. This formulation is valid for averaged, filtered and instantaneous equations as long as the correct eddy viscosity value is provided.

In multi-scale modeling, we assume the input eddy viscosity is a continuous function that varies from the RANS value to zero depending on the ratio of the grid size, $L_{G}$, to a locally defined turbulence length scale, $L_{T}$. After the appropriate eddy viscosity is found it is used in Eqs.(1) and (2). The multi-scale model allows smooth transition from RANS to LES to DNS with the grid size and local turbulent length scales being the determining factors of which model to use. When employing this approach, there are no limitations on grid size or geometry because the transition between the models is allowed to occur anywhere in the computational domain.

The multi-scale treatment can be implemented very easily into any RANS code as a mere modification to the eddy viscosity that is output from the two equation model, before using it in the mass, momentum and energy transport. This modification depends on the definition of the filter function, the turbulence length scale and the multi-scale eddy viscosity. 
The filter-based model (FBM) developed by Johansen et al. ${ }^{29}$ for implementation with the $k$ - $\varepsilon$ equations can be easily extended to the Menter's SST ${ }^{7}$ turbulence model by defining the turbulent length scale,

$$
L_{T}=\frac{\sqrt{k}}{C_{4} \max \left(\omega, S F_{2} / a_{1}\right)}
$$

the filter function,

$$
f_{d}=\min \left(1, \frac{L_{G}}{L_{T}}\right)
$$

and the eddy viscosity follows,

$$
v_{t}=v_{t_{\text {RANS }}} f_{d}
$$

Johansen et al. ${ }^{29}$ suggest setting $C_{4}$ based on $C_{\mu}$ and the anisotropy factor, $\gamma$. To avoid ambiguities with the anisotropy factor value, in this effort we relate $C_{4}$ to the Smagorinsky constant in the LES limit. First we set the eddy viscosity (away from the wall) equal to its Smagorinsky counterpart,

$$
v_{t_{\text {RANS }}} f_{d}=C_{4} L_{G} \sqrt{k}=\left(C_{s} L_{G}\right)^{2} S
$$

Assuming equilibrium between turbulence production and dissipation in the LES limit, one can approximate,

$$
\sqrt{k} \approx \sqrt{\frac{v_{t} S}{0.3}}=\frac{C_{4} L_{G} S}{0.3}
$$

Then

$$
C_{4}=\frac{C_{s}}{\sqrt{0.3}}
$$

The value of $C_{s}$ varies, but in this approach we use the value of 0.01 as recommended by Boles et al. ${ }^{20}$ for this particular case.

\section{Multi-scale hybrid RANS/LES turbulence model}

The implementation of a multi-scale turbulence model is based on the work of Nichols and Nelson ${ }^{24}$. Their $L_{\mathrm{T}}$ redefined in $k-\omega$ variables is,

$$
L_{T_{-} M S}=\max \left(6 \sqrt{\frac{v_{t_{\text {RANS }}}}{\Omega}}, \frac{k}{C_{4} \omega}\right)
$$

Where the first length scale is based on LES algebraic models and the second one is similar to FBM. We replaced $C_{\mu}$ with $C_{4}$ to be consistent with the FBM for further comparison. Their filter function uses the hyperbolic tangent for smooth transition between the models and is defined as follows,

$$
f_{d_{-} M S}=\frac{1}{2}\left\{1+\tanh \left[2 \pi\left(\frac{1}{1+\left(\frac{L_{T}}{2 L_{G}}\right)^{4 / 3}}-0.5\right)\right]\right\}
$$

The blended eddy viscosity is then defined as,

$$
v_{t_{-} M S}=v_{t_{\text {RANS }}} f_{d_{-} M S}+\left(1-f_{d_{-} M S}\right) C_{4} L_{G} \sqrt{f_{d_{-} M S} \cdot k}
$$

Where $C_{4}$ is the constant in Eq.(9) instead of the one originally used ${ }^{24}$ consistent with above FBM analysis. We can further manipulate Eq.(12) to be in the same form Eq.(6) for direct comparison with FBM,

American Institute of Aeronautics and Astronautics 
where

$$
v_{t_{-} M S}=v_{t_{\text {RANS }}} f_{d_{-} M S}^{*}
$$

$$
f_{d_{-} M S}^{*}=f_{d_{-} M S}+f_{d_{-} F B M} \sqrt{f_{d_{-} M S}}\left(1-f_{d_{-} M S}\right)
$$

Filter function role and solution dynamics

The filter functions, $f_{d-\mathrm{FBM}}, f_{d-M S}$, and $f_{d-M S}{ }^{*}$, are plotted in Figure 1 versus $L_{\mathrm{T}} / L_{\mathrm{G}}$. The filter value is near unity where $L_{\mathrm{T}} / L_{\mathrm{G}}$ is smaller than one so that the model renders standard RANS in grid areas that cannot resolve further turbulent structure. The filter also approaches zero as $L_{\mathrm{T}} / L_{\mathrm{G}}$ goes to infinity and turbulence modeling is no longer needed. The filter functions are intended to be general and capable of handling a wide range of grid resolutions and flow conditions. Therefore, in order to evaluate the filter functions in Figure 1 we must first understand their dynamic role in progressing the solution in regions varying in grid size and turbulence intensity. In all the following cases we assume that we start applying multi-scale approach to a converged RANS solution:

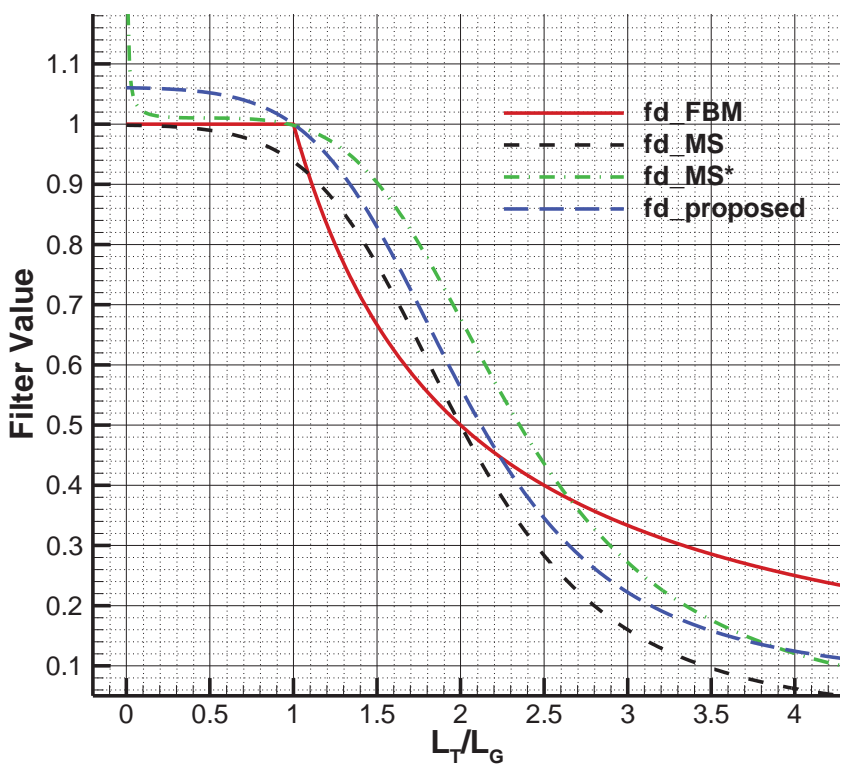

Figure 1. Filter functions for FBM, multi-scale models and proposed filter

1. Flow is approaching laminar, $L_{\underline{T}} \underline{L}_{G} \sim \infty$ : In this case the grid size can handle the flow and the filter function should approach zero. Reducing $v_{\mathrm{t}}$ will not cause any production of turbulent structures. Because the eddy viscosity is used again in the $\mathrm{k}$ transport equation. It will be recursively reduced without a stop because $L_{\mathrm{T}} / L_{\mathrm{G}}$ will not reach unity.

2. Flow is turbulent but grid is capable of resolving all turbulent scales, $\underline{L}_{\underline{T}} / \underline{L}_{\underline{G}}>1$ : In this case the flow is initially free of turbulent structures because of the original RANS solution. $f d$ will be less than 1 , and as $v_{\mathrm{t}}$ decreases more vortical structures are formed, and the RANS equations act as a subgrid model for the unresolved part of the grid. This in turns decreases $v_{\mathrm{t} \_\mathrm{R} A N S}$ and $L_{\mathrm{T}} / L_{\mathrm{G}}$ which increases the filter function as more scales are resolved. Even after all scales are resolved $L_{\mathrm{T}} / L_{\mathrm{G}}$ remains greater than 1 and $f d$ will be less than unity. Lowering the eddy viscosity further will not affect the solution negatively therefore to be successful in this region $f d$ should be low enough that it does not reach unity before all scales are resolved.

3. Flow is turbulent but grid is capable of resolving some turbulent length scales, and the unresolved portion is in the dissipation range, $L_{\underline{T}} \underline{L}_{\underline{G}}>1$ then $\underline{L}_{\underline{T}} / \underline{L}_{\underline{G}}<1$ : This represents the LES limit. The flow is initially free of turbulent structures and vortices are forming while $v_{\text {t_RANS }}$ decreases and $f d$ increases until it reaches 1 ) 
and that stops any further decrease of $v_{t}$. The value of the filter function near $f d=1$ is very critical as it determines at which value of $L_{\mathrm{T}} / L_{\mathrm{G}}$ the filtering action stops. In the case of FBM, $\mathrm{C}_{4}$ is calibrated to match the LES limit in this region which should adjust the filtering process accordingly. This however may not be an exact match as different two equation models calculate RANS variables differently. If $v_{\mathrm{t}}$ were to drop further below the LES value the vortical structures will continue to be produced until the numerical viscosity takes over and stops further eddies from forming. This will be equivalent to the Implicit LES approach.

On way to alleviate this problem is to set a lower limit for the eddy viscosity as follows:

$$
v_{t}=\max \left(v_{t}, v_{t_{-} L E S}\right)
$$

where $v_{\text {t_LES }}$ is calculated using the standard Smagorinsky model in Eq.(3).

4. Flow is turbulent but grid is capable of resolving some turbulent length scales, and the unresolved portion is not fully in the dissipation range, $\underline{L}_{\mathrm{T}} / \underline{L}_{\underline{G}}>1$ then $L_{T} / \underline{L}_{\underline{G}}<1$ : This is the hardest region to resolve because it lies between RANS and LES. There is a dependence on the filter function value at $f d=1$ as it determines when to stop the filtering process. In this region, care must be taken so that $v_{t}$ is not decreased to the implicit LES limit, and that the two equation model is used as a subgrid model for the entire process. Increasing $L_{\mathrm{T}} / L_{\mathrm{G}}$ at $f d=1$ would drive the modeling away from the implicit LES limit but would compromise resolving vortical structures at finer, higher quality grid regions. The filter function should be constructed that there is some compromise where finer regions may not resolve all possible turbulent length scales, and bad quality cells that are far from regions of interest will end up at the implicit LES limit because of the higher numerical viscosity they posses.

When there is a large variation in grid size in this region transfer of turbulent kinetic energy between the resolved and the subgrid scales may need special attention. Johansen et al. ${ }^{29}$ suggest adding an extra term to the $\mathrm{k}$ equation containing the gradient of cell size to alleviate this problem.

5. Flow is turbulent but grid is not capable of resolving any turbulent length scales, $L_{\underline{T}} / \underline{L}_{\underline{G}}<1$ : This is the RANS limit where the filter value should be very close to 1 so that it does not alter the original RANS eddy viscosity. $v_{\mathrm{t}}$ and $f d$ stay constant because no eddies are produced.

Considering the scenarios mentioned above the filter function should match $f d_{-F B M}$ as much as possible near $f d=1$ so that $C_{4}$ is calibrated correctly. $f d$ should be very close to 1 in the RANS limit when $L_{\mathrm{T}} / L_{\mathrm{G}}<=1$ with the maximum value being a little above unity to ensure that it will numerically stop reducing $v_{t}$ when it is required to do so. The filter should also be smooth for stability reasons.

From the filters in Figure 1, $f d_{-\mathrm{FBM}}$ lacks smooth transition and its maximum value is $1 . f d^{*}{ }_{\text {-MS }}$ is smooth but it posses higher filter values near $L_{\mathrm{T}} / L_{\mathrm{G}} \sim 1$ than those of $f d_{\text {-FBM }}$ and increases the RANS value by $18 \%$ as $L_{\mathrm{T}} / L_{\mathrm{G}}$ approaches zero. $f d_{-\mathrm{MS}}$ is the best choice of all three but needs higher filter values near $L_{\mathrm{T}} / L_{\mathrm{G}}<1$. A proposed filter function that is similar to $f d_{\text {-MS }}$ but shifted up so that $L_{\mathrm{T}} / L_{\mathrm{G}}=1$ at $f d=1$ is also plotted in Figure 1 . This filter is implemented by using the Nichol and Nelson's multi scale model ${ }^{24}$ with $f d^{*}{ }_{\text {-MS }}$ replace by $f d_{\text {-Proposed }}$

$$
f d_{\text {proposed }}=f d_{M S}+0.0622
$$

Note that this is also equivalent to eliminating the second term in Eq.(12), and modifying $f d_{-m s}$. 


\section{Results and Discussion}

Lin et al. ${ }^{21}$ performed a sonic injection of ethylene into Mach 2 air crossflow at the continuous flow supersonic tunnel at Wright-Patterson Air Force Base, Ohio. The tunnel has a constant test are of $131 \times 152 \mathrm{~mm}$. The injectors were circular with different diameters, injection angles, and momentum ratios. In this study we focus on the case with $D=0.1875$ inches normal injection with a momentum ratio of 0.5 . Lin et al. ${ }^{21}$ used Raman scattering technique to collect quantitative concentration data. These measurements were used to calculate the mixture fraction at various $x / D$ locations downstream of the injector.

\section{Grid and numerical choices}

RANS simulations were conducted using a 600,000 structured grid with symmetry boundary condition. This was done because the solution reached grid independence and larger grids showed similar results. For the multi-scale simulations three different grids were used with mixed structured domains as shown in Figure 2 and 3. A coarse grid contains about 7 million cells with the jet region at a finer resolution and a boundary layer grid starting at $\boldsymbol{y}^{+} \sim 10$. Intermediate grid was adapted from the coarse grid based on the filter function and ethylene mole fraction and contains about 17 million cells. A fine grid was adapted from the intermediate grid and contains 27 million cells.

Loci-Chem ${ }^{26,27}$ which is an unstructured density-based multi-species general code was used with the necessary implementations for the current effort. The inlet to the computational domain was taken from a RANS solution of a separate simulation of the nozzle. The full width and height of the wind tunnel was used in the computation. The injector geometry was also representative of that used in the experiment. Conditions of the simulation are shown in Table 1.

The simulations were run with the Menter SST turbulence model ${ }^{7}$ until converged to a RANS solution. The multi-scale approach was then applied and instantaneous results were collected and averaged.

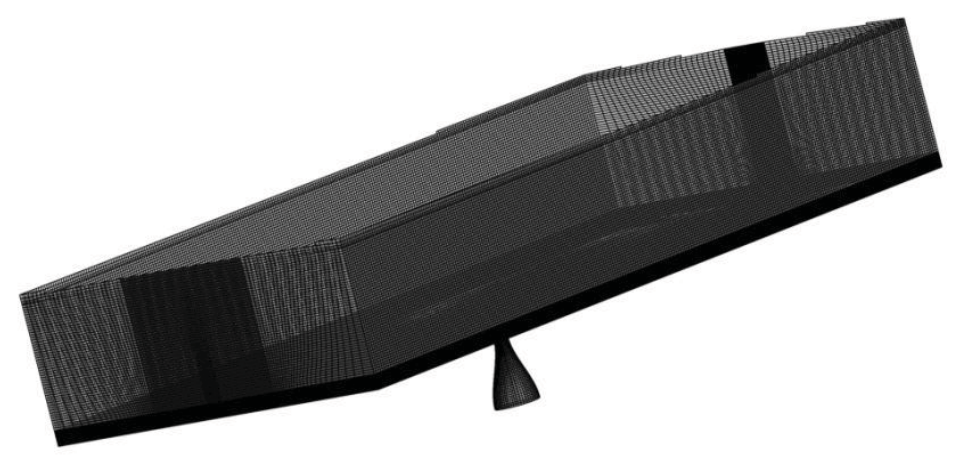

Figure 2. Coarse grid cell distributions for multi-scale simulation
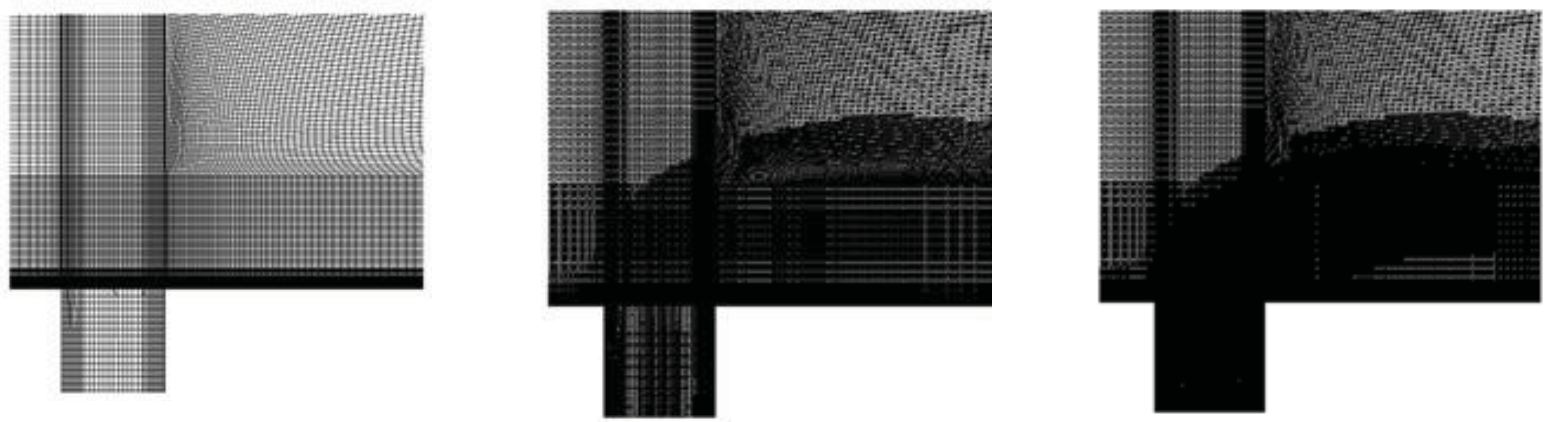

Figure 3. Center plane cut. Coarse, intermediate, and fine grids 
Table 1. Flow and geometric conditions for Ethylene injection

\begin{tabular}{c|c}
\hline Parameters & Values \\
\hline $\mathrm{P}_{0}$ (freestream) & $244 \mathrm{KPa}$ \\
$\mathrm{T}_{0}$ (freestream) & $300 \mathrm{~K}$ \\
$\mathrm{M}$ (freestream) & 2 \\
$\mathrm{P}_{0}$ (injectant) & $127.5 \mathrm{KPa}$ \\
$\mathrm{T}_{0}$ (injectant) & $322 \mathrm{~K}$ \\
$\mathrm{D}$ & $4.8 \mathrm{~mm}$ \\
$\mathrm{q}$ & 0.5 \\
Injectant angle $(\theta)$ & $90^{\circ}$ \\
\hline
\end{tabular}

\section{A. RANS solution with various turbulent Schmidt numbers}

RANS simulations without multi-scale modeling showed results that were of a different fuel structure than of the experiment consistent with the findings of other researcher ${ }^{20,25}$ who conducted the same simulation with various turbulence models and grid resolutions. In this effort, we run the simulations with Menter SST turbulence model with 3 different turbulent Schmidt numbers. Turbulent Schmidt number directly affect turbulence mixing and RANS crossflow simulations were shown to be very sensitive to their values ${ }^{17}$.

Figure 4 shows the results for three different $\mathrm{Sc}_{\mathrm{t}}(0.9,0.4$, and 0.1$)$ as well as experimental measurements. For the large $\mathrm{Sc}_{\mathrm{t}}$, results similar to other researchers ${ }^{20,25}$ were observed. Near field at $x / D=5$, the fuel is detached from the wall with an inverse heart shape. There is a large concentration of fuel in two kidney shaped vortices in the center. Then at $x / D=10$ the fuel shifts from the wall and high concentration of fuel is lifted upward leading to high penetration heights and complete detachment at $x / D=25$. When $\mathrm{Sc}_{\mathrm{t}}$ is decreased to match the fuel concentration of the experiment better results are obtained. For $\mathrm{Sc}_{\mathrm{t}}=0.4$ there is a decrease in the fuel concentration at $x / D=5$ however the two kidney vortices are still present and the fuel structure is still similar to $\mathrm{Sc}_{\mathrm{t}}=0.9$ for both $x / D=10$ and $x / D=25$. When $\mathrm{Sc}_{\mathrm{t}}=0.1$, the fuel concentration is of roughly the same values as the experiment. At $x / D=5$, the fuel core does not show the fuel rich vortex pair, however the structure still resembles an inverse heart shape and the penetration height is over predicted. At $x / D=10$ the fuel core is attached to the wall similar to the experiment however it is more elongated with penetration height and width larger than the experiment. At $x / D=25$ the fuel core is very large and dispersed showing similarities to the experiment but with larger cross sectional area.

From the results it is evident that the RANS approach with typical values of $\mathrm{Sc}_{\mathrm{t}}(0.4-0.9)$ is inadequate and shows poor correlation with the experimental measurements. It is possible to lower $\mathrm{Sc}_{\mathrm{t}}$ to match experimental fuel concentration, but the results still show different fuel structure with penetration height mismatch. The use of a constant $\mathrm{Sc}_{\mathrm{t}}$ may therefore be inappropriate. 

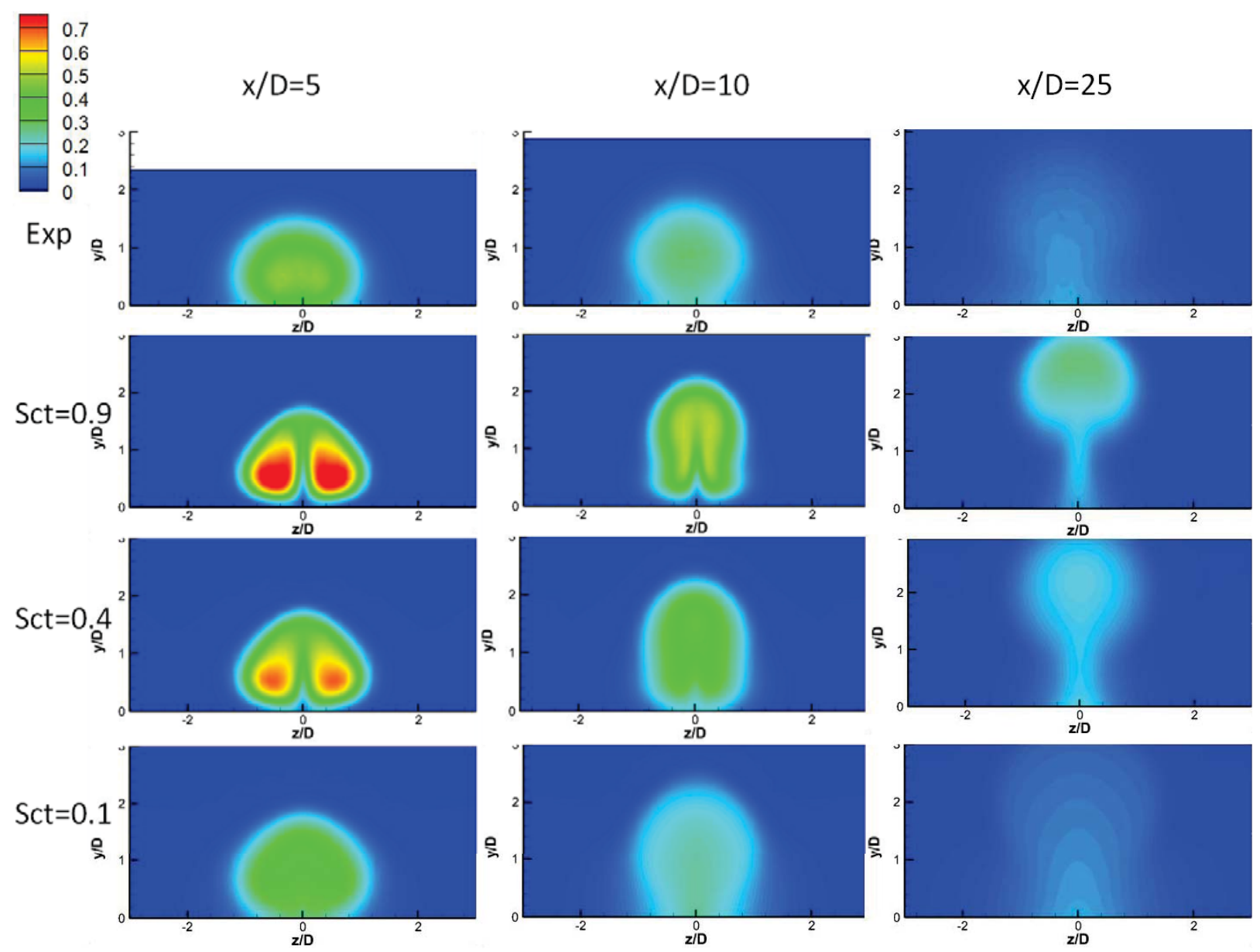

Figure 4. Average Ethylene mole fraction results for RANS with different turbulent Schmidt numbers

\section{B. Simulations with the multi-scale model}

\section{Effect of grid resolution}

Instantaneous ethylene mole fraction contours shows more detailed structures of the highly turbulent field as the grid is refined. Figure 5 shows that there are smaller and more refined fuel structures in the intermediate grid than of the coarse grid. The fine grid shows even more turbulent structures near the tip of the fuel plume where it breaks violently into smaller eddies. Figure 6 shows the instantaneous (modeled) eddy viscosity contours for all three grids. As the grid gets finer the modeled eddy viscosity becomes lower with the coarse grid showing eddy viscosities up to an order of magnitude higher than that of the fine grid.

Figure 7 shows time-averaged ethylene mole fractions collected from the multi-scale simulation. For $x / D=5$, the penetration height is well predicted for all three grids. As the grid is refined the shape of the fuel cross section becomes more flat shifting away from the "heart" shaped RANS results with more interaction with the wall boundary layer. Coarse grid shows two high fuel concentration kidney shaped vortices at the center similar to the RANS solution except that they are closer together and of less intensity. This may show a crossover between RANS and multi-scale modeling since the grid is not highly refined. Intermediate and fine grids show less concentration of the fuel center with the shape becoming more representative of the experimental results. The fuel core center is however still over predicted in the finest grid level. This may mean we need finer grid or higher order flux functions. For $x / D=10$, there is an over prediction of the penetration height similar to the RANS solution but improving as the grid refined. The solution resembles the experimental results as the grid is refined and the fuel core center becomes less concentrated. The shape of the fuel core also becomes more circular and closer to the wall. For $x / D=25$, there is also an improvement with the fuel core becoming attached to the wall resembling the experiment as the grid is refined. From the results it is evident that when more turbulent structures are physically resolved the solution 
improves. This would point to inadequate modeling of mass transport and mixing in the RANS approach which is not solved by simply reducing the turbulent Schmidt number as shown in the last section.

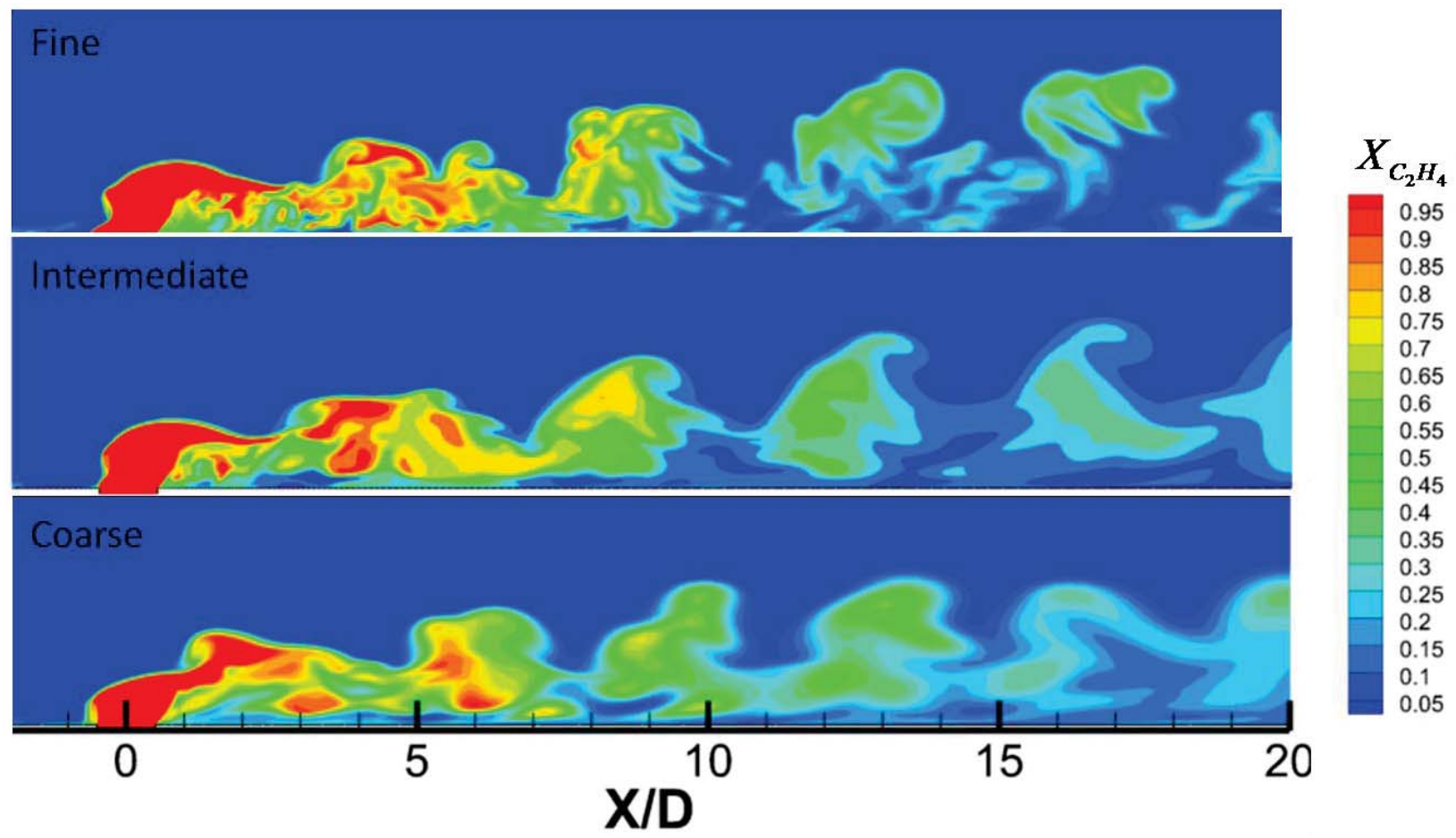

Figure 5. Instantaneous snapshots of Ethylene mole fraction contours for coarse, intermediate, and fine grids

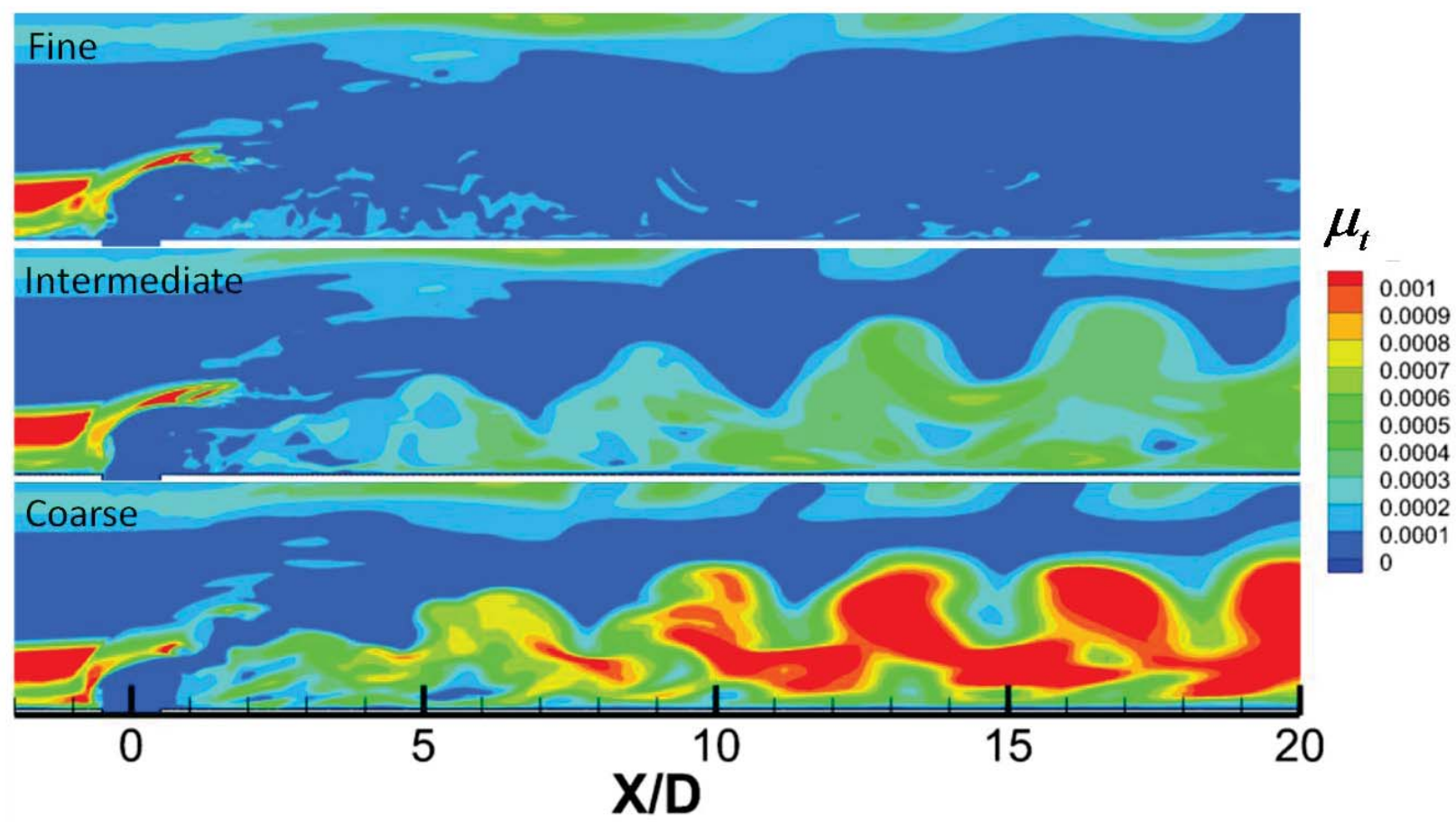

Figure 6. Instantaneous snapshots of eddy viscosity contours for coarse, intermediate, and fine grids 

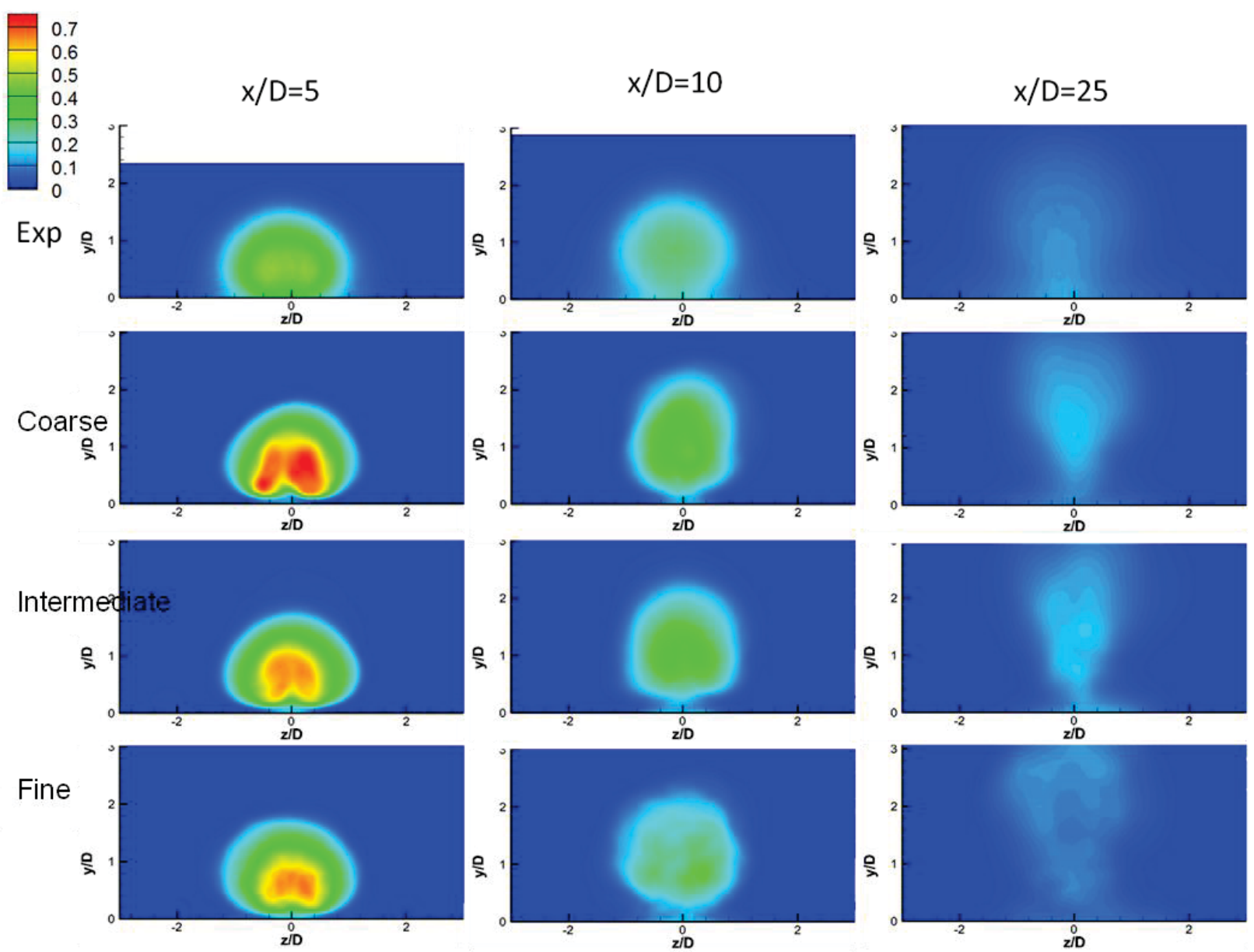

Figure 7. Average Ethylene mole fraction results for grid refinement study

Analysis of resolved turbulence for the intermediate grid

For the intermediate grid, turbulent statistics were collected to estimate turbulence kinetic energy, mass and momentum eddy viscosities, and turbulent Schmidt number based on actual fluctuations of the resolved field. The procedure used here is the one detailed in Boles et al. ${ }^{20}$ and briefly explained below.

A flux a variable $\phi$ 'may be expressed as

$$
\overline{\rho u_{j}^{\prime} \phi^{\prime}}=\overline{\rho u_{j} \phi}-\frac{\overline{\rho u_{j}} \overline{\rho \phi}}{\bar{\rho}}
$$

where the over bars mean time averages. For turbulent mass transport, the value above may be corrected with a gradient-diffusion assumption.

$$
\overline{\rho u_{j}^{\prime} Y^{\prime}}=-\bar{\rho} v_{t, \text { mass }, j} \frac{\partial \tilde{Y}}{\partial x_{j}}
$$

where a Favre-averaged variable $\tilde{\phi}$ is defined as:

$$
\tilde{\phi}=\frac{\overline{\rho \phi}}{\bar{\rho}}
$$


In general, separate eddy viscosities $v_{t, \text { mass }, j}$ could be estimated for each component of the gradient vector. The validity of the gradient-diffusion assumption requires that $\overline{\rho u_{\jmath}^{\prime} Y^{\prime}}$ be well correlated with the gradient in mole faction. Boles has verified that this is the case for the mixing region downstream of the injection at least for the $\mathrm{y}$ and $\mathrm{z}$ directions $^{18}$. As RANS model will typically use an isotropic eddy viscosity, $\mathrm{Eq}(18)$ can be written as $\overline{\rho u_{j}^{\prime} Y^{\prime}}=$ $-\bar{\rho} v_{t, m a s s}\left(\partial \tilde{Y} / x_{j}\right)$, and an effective isotropic eddy viscosity associated with mass transport can be determined by taking an $L_{2}$ norm of the turbulent diffusion velocity vector:

$$
v_{t, \text { mass,Res }} \approx-\frac{\overline{\rho u_{i}^{\prime} Y^{\prime}}}{\bar{\rho}} \frac{\left(\partial \tilde{Y} / \partial x_{i}\right)}{\left(\partial \tilde{Y} / \partial x_{i}\right)\left(\partial \tilde{Y} / \partial x_{i}\right)}
$$

Similarly, one can define isotropic eddy viscosity associated with momentum transports as follows:

$$
\begin{aligned}
& v_{t, \text { mass,Res }} \approx-\frac{\overline{\rho u_{i}^{\prime} u_{j}^{\prime}}-\left[2 / 3 \delta_{i j} \overline{\rho k}\right] S_{i j}\left(\tilde{u}_{i}\right)}{\bar{\rho} S_{i j}\left(\tilde{u}_{i}\right) S_{i j}\left(\tilde{u}_{i}\right)} \\
& \left.S_{i j} \tilde{u}_{i}\right)=\frac{\partial \tilde{u}_{i}}{\partial x_{j}}+\frac{\partial \tilde{u}_{j}}{\partial x_{i}}-\frac{2}{3} \frac{\partial \tilde{u}_{k}}{\partial x_{k}} ; \overline{\rho k}=\frac{1}{2}\left(\overline{\rho u_{k} u_{k}}-\frac{\overline{\rho u_{k}} \overline{\rho u_{k}}}{\bar{\rho}}\right)
\end{aligned}
$$

Turbulent Schmidt numbers $\left(\mathrm{Sc}_{\mathrm{t}}\right)$ may then be calculated by taking ratios of these isotropic eddy viscosities:

$$
\mathrm{Sc}_{\mathrm{t}}=\frac{v_{t, \text { mom, Res }}}{v_{t, \text { mass }, \text { Res }}} \text {. }
$$

Figure 8 shows contours of turbulence kinetic energy $K_{\text {Res, }}$ and the ratio between unresolved and total turbulence kinetic energy at the center plane. $K_{\text {Res }}$ displays a highly concentrated source just downstream of the barrel shock then diffuses quickly into the jet. At this location about 10 to $20 \%$ of the total turbulence kinetic energy is modeled that is likely responsible for the modeled eddy viscosity difference between the intermediate and fine grids further downstream that was shown in Figure 6. The plot generally demonstrated that simulation with multi-scale turbulence model with the intermediate grid was able to resolve most of the turbulent structures.

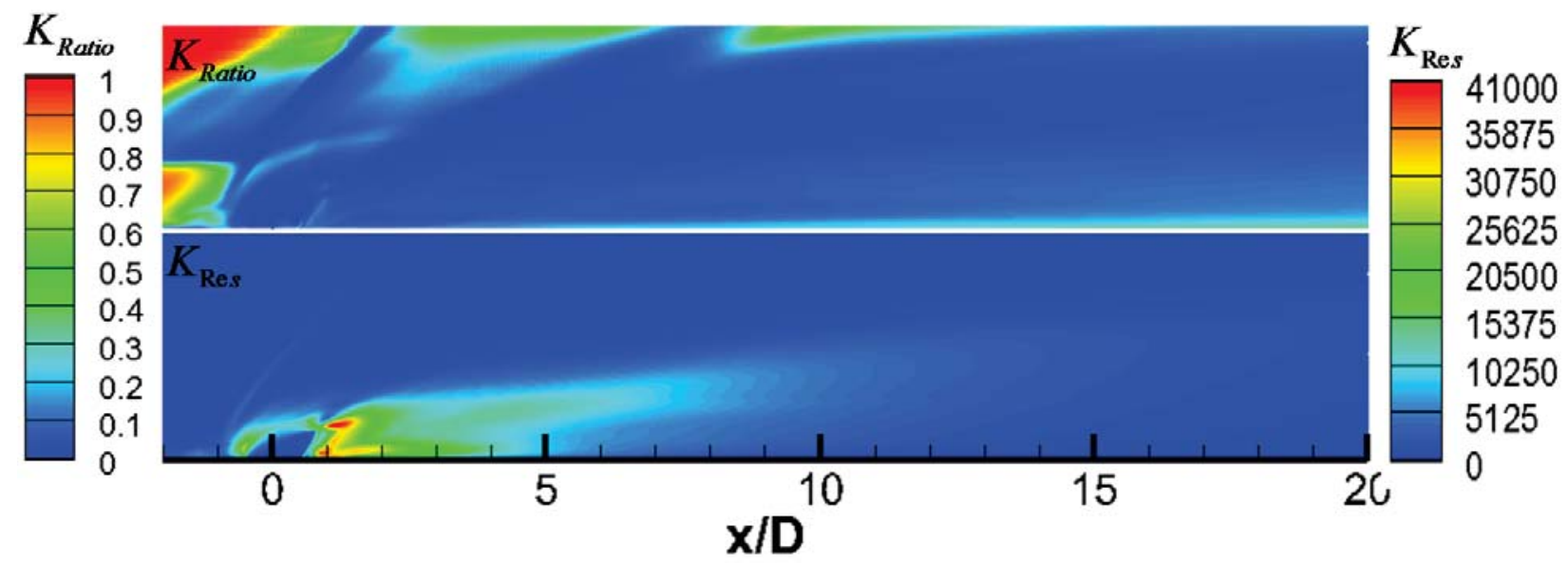
Figure 8. Turbulence kinetic energy $\left(K_{\text {Res, }}\right.$, bottom) and ratio of unresolved turbulence kinetic energy to
total turbulence kinetic energy $\left(K_{\text {Ratio }}\right.$, top)

Figure 9 shows mass and momentum eddy viscosities estimated by Eqs (20) and (21). Higher values of mass eddy viscosities are found in the center of the jet between $x / D=7$ and $x / D=15$. This is the area where the jet widens and mass is diffused outwards.. Momentum eddy viscosity shows a similar trend with high momentum fluxes in the center/upper region of the jet between $x / D=10$ and $x / D=20$. Thin layers of negative momentum eddy viscosity exist below high concentration regions similar to the mass eddy viscosity. There is however a narrow region of negative mass and momentum eddy viscosities which would translate into mass/momentum being drawn into regions of 
higher fuel mass/momentum concentration. While this is physically possible, it is unlikely because the thin region is probably the result of errors in the estimation method due to the assumption of isotropy.

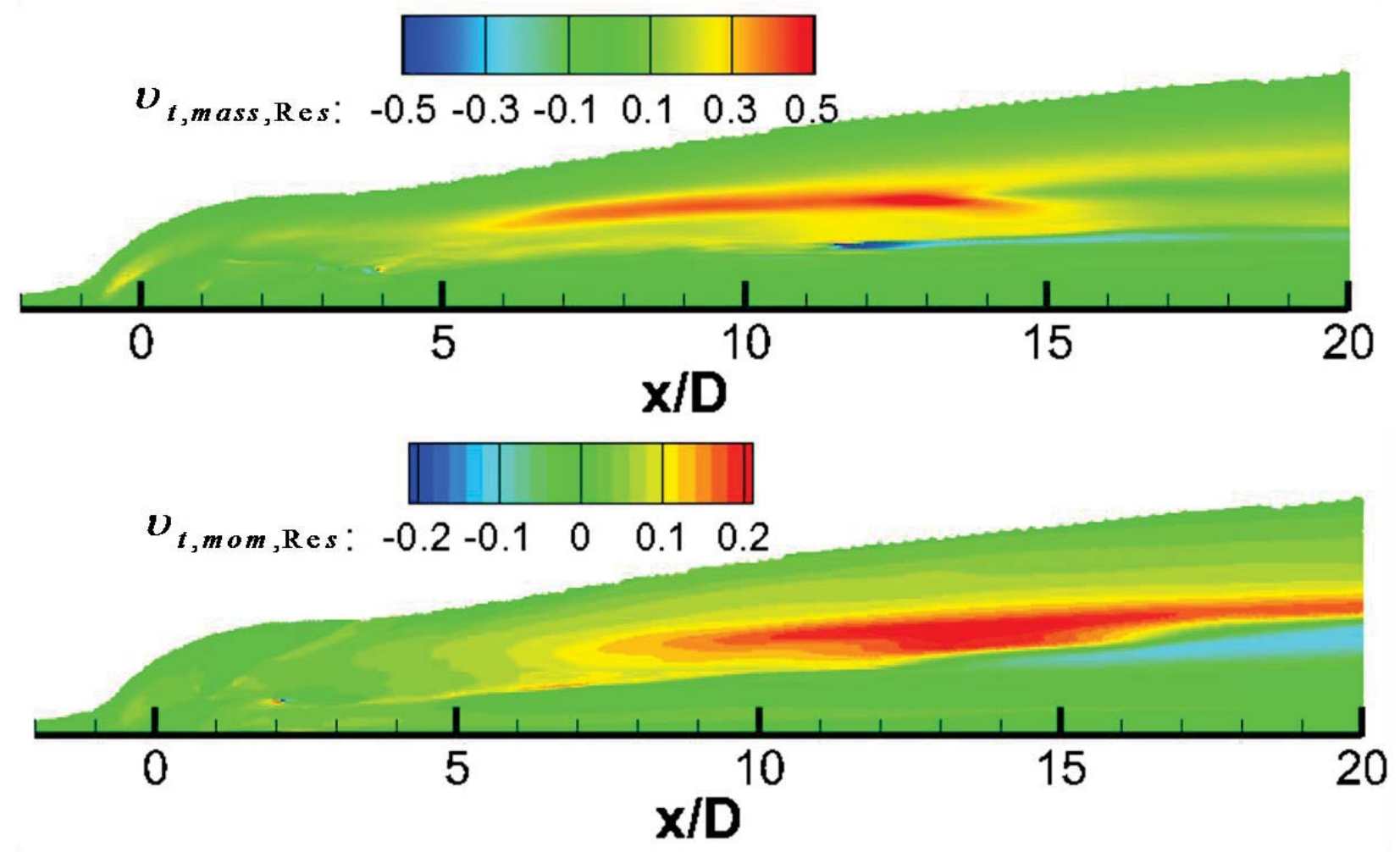

Figure 9. Mass and momentum eddy viscosities based on resolved field at the center plane

Figure 10 shows mass and momentum eddy viscosities and turbulent Schmidt number computed by Eqs (20), (21), and (22) for $x / D=5$. At the center of the jet cross section, there is a high concentration of mass and momentum eddy viscosities due to an increase of turbulent fluctuations in this region. It should be mentioned that this is the same region where the multi-scale model over predicts the fuel concentration. Therefore, finer grid or higher order fluxes maybe needed to resolve more turbulence. It is clearly observed that overall the magnitude of turbulent Schmidt number is in the lower end of its typical modeled values. It also varies depending on the location with a non uniform distribution. There are regions of negative values due to the mass and momentum eddy viscosities having opposite signs. Recalling the effects of constant turbulent Schmidt number on RANS simulations and non-uniform turbulent Schmidt number distribution on the plane obtained by current multi-scale simulations, we intend to perform simulations with the turbulent Schmidt number based on the resolved field adaptively used to model its value in the subgrid RANS equations. 

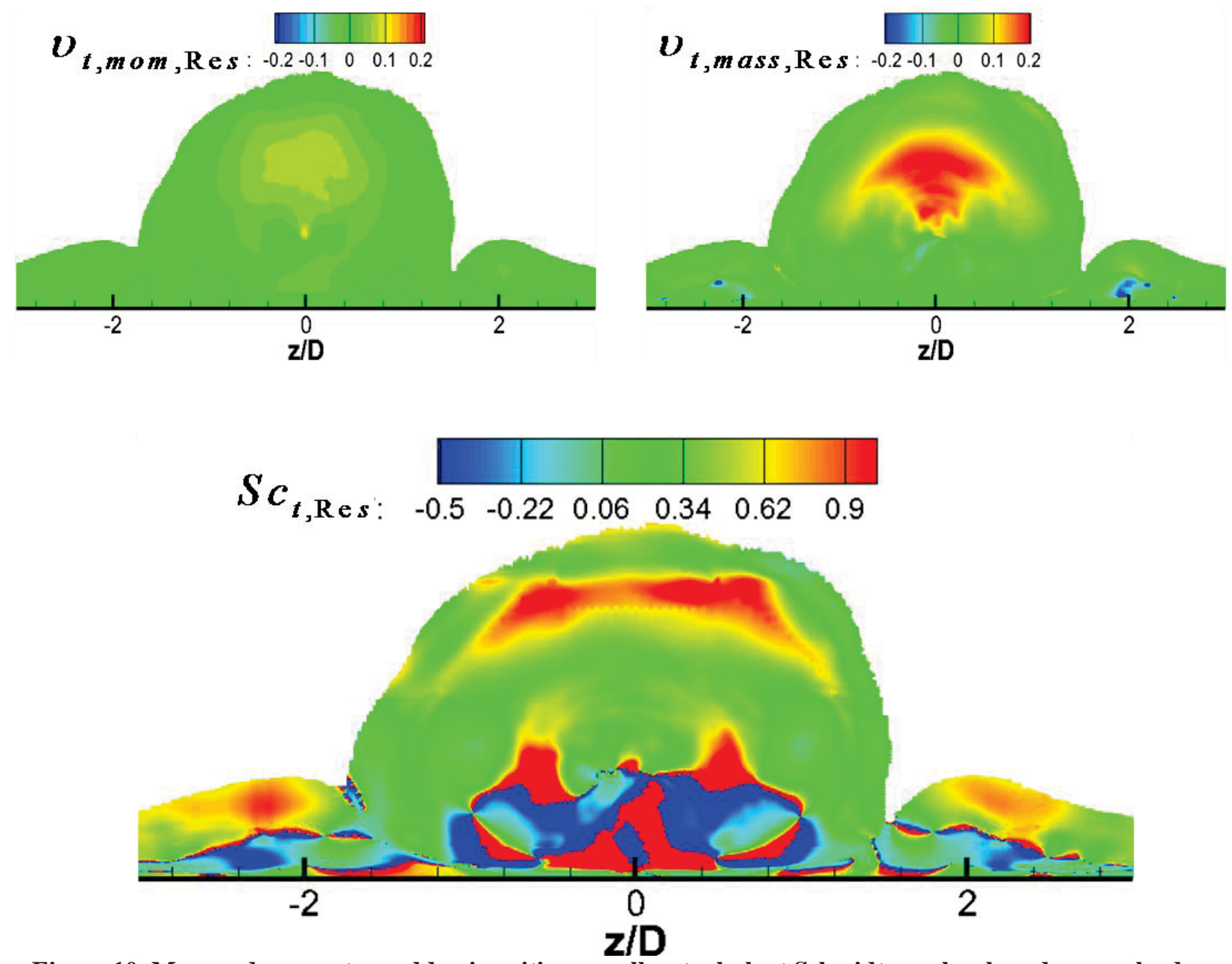

Figure 10. Mass and momentum eddy viscosities as well as turbulent Schmidt number based on resolved field for $x / D=5$

\section{Summary and Conclusions}

A multi-scale hybrid RANS/LES approach was developed and implemented to simulate a case of sonic ethylene injection into supersonic air crossflow. The approach has a unique filter function to limit the eddy viscosity in RANS simulations, resolving turbulent eddies in regions where the grid resolution is sufficient. Fuel turbulent structures were resolved with more eddies present as the grid is refined. Results with the multi-scale model showed a significant improvement over RANS when compared to experimental measurements. The results were a closer match to the experiment as the grid is adapted. RANS results on the other hand could be improved by changing the turbulent Schmidt number $\left(\mathrm{Sc}_{\mathrm{t}}\right)$, however, the results still deviated from the experiment in penetration height and width suggesting a non-constant $\mathrm{Sc}_{\mathrm{t}}$ model for RANS. Turbulent statistics and fluctuations were collected from the multi-scale simulation. Modeled turbulent kinetic energy at the regions of interest was a small portion of the total turbulent kinetic energy. Mass and momentum eddy viscosities were estimated based on the resolved field using the turbulent mass and momentum fluxes respectively. Turbulent Schmidt number based on resolved field was not at all constant and varied with most of the domain at lower values. In future work, the turbulent Schmidt number predicted can be used to adaptively change its value in the subgrid RANS model.

\section{Acknowledgements}

Ez A Hassan would like to acknowledge the SMART program for their support. Portions of the computations were performed on AFRL high performance computing clusters. 


\section{References}

1. Santiago, J. G., and Dutton, J. C. "Velocity Measurements of a Jet Injected into a Supersonic Crossflow," Journal of Propulsion and Power Vol. 11, No. 2, 1997, pp. 315-323.

doi: $10.2514 / 2.5357$

2. $\quad$ Everett, D. E., Woodmansee, M. A., Dutton, J. C., and Morris, M. J. "Wall Pressure Measurements for a Sonic Jet Injected Transversely Into a Supersonic Crossflow," Journal of Propulsion and Power Vol. 14, No. 6, 1998, pp. 861868.

doi: $10.2514 / 2.5357$

3. VanLerberghe, W. M., Santiago, J. G., Dutton, J. C., and Lucht, R. P. "Mixing of a Sonic Transverse Jet Injected into Supersonic Crossflow," AIAA Journal Vol. 38, No. 3, 2000, pp. 470-479.

doi: $10.2514 / 2.984$

4. Gruber, M. R., Nejad, A. S., Chen, T. H., and Dutton, J. C. "Large structure convection velocity measurements in compressible transverse injection flowfields," Experiments in Fluids Vol. 22 (1997), 1997, pp. 397-407.

5. Ben-Yakar, A., Mungal, M. G., and Hanson, R. K. "Time Evolution and Mixing Characteristics of Hydrogen and Ethylene Transverse Jets in Supersonic Crossflows," Physics of Fluids Vol. 18, No. 2, Feb,2006, p. 026101. doi: 10.1063/1.2139684

6. Tam, C.-J., Baurle, R. A., and Gruber, M. R. "Numerical Study of Jet Injection into a Supersonic Crossflow," 35th AIAA/ASME/SAE/ASEE Joint Propulsion Conference and Exhibit. Vol. A99-31100, Los Angeles, california, 1999.

7. Menter, F. R. "Zonal two equations k-w turbulence models for aerodynamic flows," AIAA Vol. 93, No. $2909,1993$.

8. Wilcox, D. C. "Simulation of Transition With a Two-Equation Turbulence Model," AIAA Journal Vol. 32, 1994, pp. 247-254.

9. Gruber, M. R., Nejad, A S., J. C. Dutton. "An Experimental Investigation of Transverse Injection from Circular and Elliptical Nozzles into supersonic Crossflow," Wright Lab Technical Report Vol. WL-TR-96-2102, 1996.

10. Gruber, M. R., Nejad, A. S., Chen, T. H., and Dutton, J. C. "Compressibility Effects in Supersonic Transverse Injection Flowfields," Physics of Fluids Vol. 9, No. 5, 1997, pp. 1448-1461.

11. Palekar, A., Truman, C. R., and Vorobieff, P. "Prediction of Transverse Injection of a Sonic Jet in a Supersonic Crossflow," 36th AIAA Plasmadynamics on Lasers Conference. Vol. 2005-5366, Toronto, Ontario Canada, 2005.

12. Maddalena, L., Campioli, T. L., and Schetz, J. A. "Experimental and Computational Investigations of Light-Gas Injectors in Mach 4.0 Crossflow," Journal of Propulsion and Power Vol. 22, No. 5, 2006.

13. Nichols, R. H., and Tramel, R. "Applications of a Highlt Efficient Numerical Method for Overset Mesh Moving Body Problems," AIAA Vol. 97-2255, 1997.

14. Sinha, N., Dash, S., and Chidambaram, N. "A perspective on the Simulation of Cavity Aeroacoustics," AIAA Vol. 980286, 1998.

15. Spalart, P., Jou, W., and Strelets, M. Comments on the Feasibility of LES for Wings and ona Hybrid RANS/LES Approach: Greyden Press, Columbus, OH, 1997.

16. Kawai, S., and Lele, S. K. "Large-Eddy Simulation of Jet Mixing in Supersonic Crossflows," AIAA Journal Vol. 48, No. 9, 2010, pp. 2063-2083.

doi: $10.2514 / 1 . J 050282$

17. Peterson, D. M., Subbareddy, P. K., and Candler, G. V. "Assessment of Synthetic Inflow Generation for Simulating Injection into Supersonic Crossflow," 14th AIAA/AHI Space Planes and Hypersonic Systems and Technologies Conference. 2006.

18. Boles, J. A. "Hybrid Large-Eddy Simulation/Reynolds-Averaged Navier-Stokes Methods and Predictions for Various High-Speed Flows," Aerospace Engineering. Vol. Doctor of Philosophy, North Carolina State University, Raleigh, NC, 2009.

19. Boles, J. A., and Edwards, J. R. "Hybrid LES/RANS Simulation of Transverse Sonic Injection into a Mach 2 Flow," 46th AIAA Aerospace Sciences Meeting and Exhibit. Vol. 2008-622, Reno, Nevada, 2008.

20. Boles, J. A., Edwards, J. R., and Baurle, R. A. "Large-Eddy/Reynolds-Averaged Navier-Stokes Simulations of Sonic Injection into Mach 2 Crossflow," AIAA Journal Vol. 48, No. 7, 2010, pp. 1444-1456.

doi: 10.2514/1.J050066

21. Lin, K.-C., Ryan, M., Carter, C., Gruber, M., and Raffoul, C. "Scalability of Ethylene Gaseous Jets for Fueling HighSpeed Air-Breathing Combustors," 47th AIAA Aerospace Sciences Meeting and Exhibit. Vol. 2009-1423, Orlando, Florida, 2009.

22. Spalart, P. R., Deck, S., Shur, M. L., Squires, K. D., Strelets, M. K., and Travin, A. "A new version of detached-eddy simulation, resistant to ambigious grid densities," Theoritical Computational Fluid Dynamics Vol. 20, 2006, pp. 181195.

23. Shur, M. L., Spalart, P. R., Strelets, M. K., and Travin, A. K. "A hybrid RANS-LES approach with delayed-DES and wall modelled LES capabilities," International Journal of Heat and Fluid Flow Vol. 29, No. 6, 2008, pp. 1638-1649.

24. Nichols, R. H., and Nelson, C. C. "Application of Hybrid RANS/LES Turbulence Models," 41st Aerospace Science Meeting and Exhibit. Reno , Nevada, 2003. 
25. Peterson, D. M., and Candler, G. V. "Supersonic Combustor Fuel Injection Simulation Using a Hybrid RANS/LES Approach," 48th AIAA Aerospace Sciences Meeting Including the New Horizon Forum and Aerospace Exposition. Orlando, Florida, 2010.

26. Luke, E. A., and Cinnella, P. "Numerical simulations of mixtures of fluids using upwind algorithms," Computers \& Fluids Vol. 10, 2007, pp. 1547-1566.

27. Luke, E. A., and George, T. "Loci: A rule-based framework for parallel multidisciplinary simulation synthesis," Journal of Functional Programming Vol. 15, No. 3, 2005, pp. 477-502.

28. Roe, P. L. "Approximate Riemann solvers parameter vectors and difference schemes," Journal of Computational Physics Vol. 43, 1981, pp. 357-372.

29. Johansen, S. T., Wu, J., and Shyy, W. "Filter-based unsteady RANS computations," International Journal of Heat and Fluid Flow Vol. 25(2004), 2004, pp. 10-21. 